\title{
THE MODULUS OF OPERATORS ON GROUP ALGEBRAS
}

\author{
ALI. GHAFFARI
}

\begin{abstract}
Let $G$ be a locally compact group. In this paper, we study the modulus of right multipliers on second dual of group algebras and modulus of operators on $L^{\infty}(G)$ which commute with convolutions.
\end{abstract}

\section{Preliminaries and Notations}

Let $G$ be a locally compact group. For $f \in L^{\infty}(G)$ and $\mu \in L^{1}(G)$, let the functional $f \mu \in L^{\infty}(G)$ be defined by $\langle f \mu, \nu\rangle=\langle f, \mu * \nu\rangle$ where $\nu \in L^{1}(G)$. Also for $F \in L^{1}(G)^{* *}$, let $F f \in L^{\infty}(G)$ be defined by $\langle F f, \mu\rangle=\langle F, f \mu\rangle$. Finally for $F, G \in L^{1}(G)^{* *}$, let $F G \in$ $L^{1}(G)^{* *}$ be defined by $\langle F G, f\rangle=\langle F, G f\rangle$. We know that $L^{1}(G)^{* *}$ with the first Arens product defined as above is a Banach algebra. Also, we can define the first Arens product on $L U C(G)^{*}$ by symmetry. Of course, it is well known that $L U C(G)=L^{\infty}(G) L^{1}(G)$ and $R U C(G)=L^{1}(G) L^{\infty}(G)$. If $\pi: L^{1}(G)^{* *} \rightarrow L U C(G)^{*}$ is the adjoint of embedding of $L U C(G)$ in $L^{\infty}(G)$, then for $F, G \in L^{1}(G)^{* *}$ and $f \in L^{\infty}(G)$, we have $F G=F \pi(G)$ and $F f=\pi(F) f[6]$.

For a Banach lattice $X$ and an operator $T$ on $X$, the modulus $|T|$ of $T$ is defined by $|T|(x)=\sup \{|T(y)| ;|y| \leq x\}$ for all $x \geq 0$, provided that the supremum exists ([1], [3]). Most of our notation in this paper is taken from ([3], [6]).

We say that a bounded linear map $T: L^{\infty}(G) \rightarrow L^{\infty}(G)$ commutes with convolutions if $T(f \mu)=T(f) \mu$, for $f \in L^{\infty}(G)$ and $\mu \in L^{1}(G)$. Lau and Pym in [6] have studied the operators on $L^{\infty}(G)$ which commute with convolutions. Ghahramani and Lau studied the modulus of left multipliers on $L^{1}(G)^{* *}[3]$. We prove, among other things, that if $n=\Gamma_{E}(\mu)=$ weak $^{*}$-limit $e_{\alpha} * \mu[2]$ where $\mu \in M(G)$ and $E$ is a weak ${ }^{*}$-limit of $\left(e_{\alpha}\right)$ $\left(\left(e_{\alpha}\right)\right.$ is a bounded approximate identity in $\left.L^{1}(G)\right)$, then $\left|\rho_{n}\right|(\nu)=\nu|n|$ for all $\nu \in L^{1}(G)$ (see below). Moreover, we know that if $T$ is an operator on $L^{\infty}(G)$ which commute with convolutions, then there exists $n \in L^{1}(G)^{* *}$ (or $n \in L U C(G)^{*}$ ) such that $T=T_{n}$ where $T_{n}$ is defined by $T_{n}(f)=n f$ for all $f \in L^{\infty}(G)[6]$. We show that if $\mu \in M(G)$ and $E$ is a weak*-limit of a bounded approximate identity in $L^{1}(G)$, then $\left|T_{n}\right|=T_{|n|}$ where $n=\Gamma_{E}(\mu)$.

Finally, we recall that for $\mu \in M(G)$ the functional $\mu: L U C(G) \rightarrow \mathbb{C}$ given by $\langle\mu, f \nu\rangle=\langle f, \nu * \mu\rangle$ is a member of $L U C(G)^{*}$.

2000 Mathematics Subject Classification. 43A10, 46H25.

Key words and phrases. Modulus of operators, right multipliers, convolution operators. 


\section{The Modulus of Operators}

Let $H$ be a topologically left invariant subspace of $L^{\infty}(G)$, i.e. $f \mu \in H$ for all $f \in H$ and $\mu \in L^{1}(G)$. We define $M\left(L^{\infty}(G), H\right)=\left\{T, T: L^{\infty}(G) \rightarrow H\right.$ is a bounded linear map and $T(f \mu)=T(f) \mu$ for $\left.f \in L^{\infty}(G), \mu \in L^{1}(G)\right\}$. It is known that $M\left(L^{\infty}(G), H\right)$ can be identified with a subspace of $L U C(G)^{*}[6]$. Lau and Pym have characterized $M\left(L^{\infty}(G), L U C(G)\right)$ and $M\left(L^{\infty}(G), C(G)\right)[6]$. For the subspace $H=\left\{f \in L^{\infty}(G)\right.$ : $x \rightarrow \delta_{x} f$ is weak continuous and for all $F \in L^{1}(G)^{* *}$ and $\mu \in L^{1}(G), \int\left\langle F, \delta_{x} f\right\rangle d \mu(x)=$ $\langle F \mu, f\rangle\}$ of $L^{\infty}(G)$, we characterize $M\left(L^{\infty}(G), H\right)$.

We recall that the map $\pi$ is the identity on $L^{1}(G)$, so $\pi\left(L^{1}(G) L^{1}(G)^{* *}\right)=L^{1}(G)$ $L^{1}(G)^{* *}$. Indeed, the map $\pi$ is an isometric isomorphism of $L^{1}(G) L^{1}(G)^{* *}$ onto $\pi\left(L^{1}(G)\right.$ $\left.L^{1}(G)^{* *}\right)$. Let $\tau$ be a topology on $L U C(G)^{*}$ such that $n_{\alpha} \rightarrow n$ in the $\tau$-topology if and only if for all $F \in L^{1}(G)^{* *}$ and $f \in L^{\infty}(G),\left\langle F, \delta_{x} n_{\alpha} f\right\rangle \rightarrow\left\langle F, \delta_{x} n f\right\rangle$ in the uniform topology on compacta. In the following Theorem, we will show that $\operatorname{cl}\left(L^{1}(G) L^{1}(G)^{* *}\right)=$ $M\left(L^{\infty}(G), H\right)$ where the closure is taken in the $\tau$-topology.

Theorem 2.1. Let $H$ and $\tau$ be given as above. Then

$$
\operatorname{cl}\left(L^{1}(G) L^{1}(G)^{* *}\right)=M\left(L^{\infty}(G), H\right) .
$$

Proof. It is easy to see that $L^{1}(G) \subseteq M\left(L^{\infty}(G), H\right)$, so by ([6], Lemma 3.1),

$$
L^{1}(G) L^{1}(G)^{* *} \subseteq M\left(L^{\infty}(G), H\right) .
$$

We prove that $M\left(L^{\infty}(G), H\right)$ is $\tau$-closed. Let $\left(n_{\alpha}\right)$ be a net in $M\left(L^{\infty}(G), H\right)$ such that $n_{\alpha} \rightarrow n\left(n \in L U C(G)^{*}\right)$ in the $\tau$-topology. Now if $f \in L^{\infty}(G), F \in L^{1}(G)^{* *}$ and $x_{0} \in G$, then for $\varepsilon>0$, there is a relatively compact neighbourhood $U$ of $x_{0}$ and a $\alpha_{0}$ such that for all $x \in U,\left|\left\langle F, \delta_{x} n_{\alpha_{0}} f\right\rangle-\left\langle F, \delta_{x} n f\right\rangle\right|<\varepsilon / 3$. Hence, there is a neighbourhood $V \subseteq U$ containing $x_{0}$ such that for all $x \in V,\left|\left\langle F, \delta_{x} n_{\alpha_{0}} f\right\rangle-\left\langle F, \delta_{x_{0}} n_{\alpha_{0}} f\right\rangle\right| \leq \varepsilon / 3$. Consequently, for $x \in V$ we have

$$
\begin{aligned}
\left|\left\langle F, \delta_{x} n f\right\rangle-\left\langle F, \delta_{x_{0}} n f\right\rangle\right| \leq & \left|\left\langle F, \delta_{x} n f\right\rangle-\left\langle F, \delta_{x} n_{\alpha_{0}} f\right\rangle\right|+\left|\left\langle F, \delta_{x} n_{\alpha_{0}} f\right\rangle-\left\langle F, \delta_{x_{0}} n_{\alpha_{0}} f\right\rangle\right| \\
& +\left|\left\langle F, \delta_{x_{0}} n_{\alpha_{0}} f\right\rangle-\left\langle F, \delta_{x_{0}} n f\right\rangle\right|<\varepsilon .
\end{aligned}
$$

On the other hand, for any $\alpha$ and $\mu \in L^{1}(G), \int\left\langle F, \delta_{x} n_{\alpha} f\right\rangle d \mu(x)=\left\langle F \mu, n_{\alpha} f\right\rangle$. Since $n_{\alpha} \rightarrow n$ in the $\tau$-topology, hence $\int\left\langle F, \delta_{x} n f\right\rangle d \mu(x)=\langle F \mu, n f\rangle$. It follows that $n f \in H$. So $\operatorname{cl}\left(L^{1}(G) L^{1}(G)^{* *}\right) \subseteq M\left(L^{\infty}(G), H\right)$.

To prove the reverse inclusion, let $n \in M\left(L^{\infty}(G), H\right)$ and $U$ be a compact neighbourhood of $e$ in $G$. For a bounded approximate identity $\left(e_{\alpha}\right)$ in $L^{1}(G)$ with supp $e_{\alpha} \subseteq U$, we will prove that $e_{\alpha} n \rightarrow n$ in the $\tau$-topology. If $F \in L^{1}(G)^{* *}, f \in L^{\infty}(G)$ and $K$ is a compact subset of $G$, then we can take $\psi \in C_{0}(G)$ such that $\psi(K U)=1$. For $\varphi(x)=\left\langle F, \delta_{x} n f\right\rangle$ and $\varphi_{\alpha}(x)=\left\langle F, \delta_{x} * e_{\alpha} n f\right\rangle$, we have

$$
\begin{aligned}
\left\langle\varphi, \delta_{x} * e_{\alpha}\right\rangle & =\int \varphi(t) d \delta_{x} * e_{\alpha}(t)=\int\left\langle F, \delta_{t} n f\right\rangle d \delta_{x} * e_{\alpha}(t) \\
& =\left\langle F, \delta_{x} * e_{\alpha} n f\right\rangle=\varphi_{\alpha}(x) .
\end{aligned}
$$


But for $x \in K$, we have $\left\langle\varphi \psi, \delta_{x} * e_{\alpha}\right\rangle=\left\langle\varphi, \delta_{x} * e_{\alpha}\right\rangle$. On the other hand, $\varphi \psi$ is of the form $\nu g=\varphi \psi$ for some $\nu \in L^{1}(G)$ and $g \in L^{\infty}(G)$. So for $x \in K$ we have

$$
\varphi_{\alpha}(x)=\left\langle\varphi, \delta_{x} * e_{\alpha}\right\rangle=\left\langle\varphi \psi, \delta_{x} * e_{\alpha}\right\rangle=\left\langle\nu g, \delta_{x} * e_{\alpha}\right\rangle
$$

Sicne $\left(e_{\alpha}\right)$ is an approximate identity, hence $\varphi_{\alpha} \rightarrow \varphi$ uniformly on $K$. Consequently $c l\left(L^{1}(G) L^{1}(G)^{* *}\right)=M\left(L^{\infty}(G), H\right)$.

By above Theorem, the set of all operators $T: L^{\infty}(G) \rightarrow H$ which commute with convolutions is identified with $\operatorname{cl}\left(L^{1}(G) L^{1}(G)^{* *}\right)$ (the closure is taken in the $\tau$-topology). By [6] we know that $T: L^{\infty}(G) \rightarrow L^{\infty}(G)$ commute with convolutions if and only if for some $n \in L U C(G)^{*}, T=T_{n}$ where $T_{n}(f)=n f$ for $f \in L^{\infty}(G)$. In the following Proposition, we show that if $\mu \in M(G)$ and $E$ is a weak*-limit of a bounded approximate identity in $L^{1}(G)$, then for $n=\Gamma_{E}(\mu)$ we have $\left|T_{n}\right|=T_{|n|}$. Of course, we recall that for $1 \leq p \leq \infty$, the space $L^{p}(G)$ is a complete Banach lattice with positive cone

$$
C=\left\{f \in L^{p}(G): f \geq 0 \quad \text { almost every where }\right\} .
$$

Moreover for $\mu \in M(G)$, we take $\rho_{\mu}$ as a right multiplier on $L^{1}(G)$, i.e. $\rho_{\mu}(\nu)=\nu * \mu$ for all $\nu \in L^{1}(G)$.

Proposition 2.2. The following statements hold:

(1) For $\mu \in M(G),\left|\rho_{\mu}^{*}\right|=\left.\rho_{|\mu|}\right|^{*}$.

(2) If $n=\Gamma_{E}(\mu)$, then $\left|T_{n}\right|=T_{|n|}$.

(3) If $n \in \operatorname{LUC}(G)^{\perp}$ and $n \neq 0$, then $\left|T_{n}\right| \neq T_{|n|}$ where $\operatorname{LUC}(G)^{\perp}=\left\{F \in L^{1}(G)^{* *}\right.$; $\langle F, f\rangle=0, f \in L U C(G)\}$.

Proof. Let $\mu \in M(G)$ and supp $\mu$ be compact. If $\Delta$ is a modular function of $G$, we define $\hat{\mu} \in M(G)$ by $\langle\hat{\mu}, f\rangle=\left\langle\mu,(f \Delta)^{\star}\right\rangle$, where $f \in C_{0}(G)$, and for $f \in L^{\infty}(G)$, $f^{\star}(x)=f\left(x^{-1}\right)(x \in G)$. Now for $f \in C_{0}(G)^{+}, x \in G$, we have

$$
\begin{aligned}
\left\langle|\mu|, L_{x} f\right\rangle & =\sup \left\{|\langle\mu, h\rangle| ;|h| \leq L_{x} f\right\} \\
& =\sup \left\{\left|\left\langle\mu,(h \Delta)^{\star}\right\rangle\right| ;\left|(h \Delta)^{\star}\right| \leq L_{x} f\right\} \\
& =\sup \left\{\left|\left\langle\mu,(h \Delta)^{\star}\right\rangle\right| ;|h \Delta| \leq\left(L_{x} f\right)^{\star}\right\} \\
& =\sup \left\{|\langle\hat{\mu}, h\rangle| ;|h| \leq\left(L_{x} f \Delta\right)^{\star}\right\} \\
& =\left\langle|\hat{\mu}|,\left(L_{x} f \Delta\right)^{\star}\right\rangle .
\end{aligned}
$$

Consequently,

$$
\int f(x y) d|\mu|(y)=\left\langle|\mu|, L_{x} y\right\rangle=\left\langle|\hat{\mu}|,\left(L_{x} f \Delta\right)^{\star}\right\rangle=\int f\left(x y^{-1}\right) \Delta\left(y^{-1}\right) d|\hat{\mu}|(y) .
$$

Hence for all $\nu \in L^{1}(G)$, we have $\langle|\mu| f, \nu\rangle=\langle f *|\hat{\mu}|, \nu\rangle$, i.e. $|\mu| f=f *|\hat{\mu}|$.

It is easy to see that for all $f \in L^{\infty}(G)^{+}$with compact support $\langle|\mu| f, \nu\rangle=\langle f *|\hat{\mu}|, \nu\rangle$ $\left(\nu \in L^{1}(G)\right)$. Also, it is obvious that for $g \in L^{\infty}(G)$ with compact support $\mu g=g * \hat{\mu}$. 
Now by an argument similar to the proof in ([3], Theorem 3.5), we have $\left|\rho_{\mu}^{*}\right|=\rho_{|\mu|^{*}}$ for all $\mu \in M(G)$.

2) For $f \in L^{\infty}(G)^{+}$,

$$
\left|T_{n}\right|(f)=\sup \left\{|n g| ; g \in L^{\infty}(G),|g| \leq f\right\}=\sup \left\{|E \mu g| ; g \in L^{\infty}(G),|g| \leq f\right\} .
$$

But for $g \in L^{\infty}(G)$ and $\nu \in L^{1}(G)^{+}$, we have

$$
\begin{aligned}
\langle|E \mu g|, \nu\rangle & =\sup \left\{|\langle E \mu g, \eta\rangle| ; \eta \in L^{1}(G),|\eta| \leq \nu\right\} \\
& =\sup \left\{|\langle g, \eta * \mu\rangle| ; \eta \in L^{1}(G),|\eta| \leq \nu\right\}=\left\langle\left|\rho_{\mu}^{*}(g)\right|, \nu\right\rangle .
\end{aligned}
$$

So, $|E \mu g|=\left|\rho_{\mu}^{*}(g)\right|$. Consequently,

$$
\left|T_{n}\right|(f)=\sup \left\{\left|\rho_{\mu}^{*}(g)\right| ; g \in L^{\infty}(G),|g| \leq f\right\}=\left|\rho_{\mu}^{*}\right|(f) .
$$

But by $(1),\left|\rho_{\mu}^{*}\right|(f)=\rho_{|\mu|} *(f)=|\mu| f$, so $\left|T_{n}\right|(f)=|\mu| f$. On the other hand, $\pi(|n|)=|\mu|$. Indeed, for $f \in L^{\infty}(G)^{+}$and $\nu \in L^{1}(G)^{+}$,

$$
\langle\nu \pi(|n|), f\rangle=\langle\nu|n|, f\rangle=\langle|n|, f \nu\rangle=\sup \left\{|\langle n, g\rangle| ; g \in L^{\infty}(G),|g| \leq f \nu\right\} .
$$

Now, if $g \in L^{\infty}(G)$ and $|g| \leq f \nu$, we have $|\langle n, g\rangle|=\left|\lim \left\langle e_{\alpha} * \mu, g\right\rangle\right| \leq \lim \left|\left\langle e_{\alpha} * \mu, g\right\rangle\right| \leq$ $\langle|\mu|, f \nu\rangle=\langle\nu *|\mu|, f\rangle$. Consequently, $\nu \pi(|n|) \leq \nu *|\mu|$. But by ([3], Theorem 3.1) $\rho_{|\mu|}=\left|\rho_{\mu}\right|$, so

$$
\begin{aligned}
\nu *|\mu| & =\rho_{|\mu|}(\nu)=\left|\rho_{\mu}\right|(\nu)=\sup \left\{|\eta * \mu| ; \eta \in L^{1}(G),|\eta| \leq \nu\right\} \\
& =\sup \left\{|\eta n| ; \eta \in L^{1}(G),|\eta| \leq \nu\right\} \leq \nu|n|=\nu \pi(|n|) .
\end{aligned}
$$

It follows that for all $\nu \in L^{1}(G)$ and $\nu \geq 0$, we have $\nu|n|=\nu *|\mu|$. Therefore $\pi(|n|)=|\mu|$.

3) Let $n \in L U C(G)^{\perp}$ and $n \neq 0$. For $f \in L^{\infty}(G)$ with $f \geq 0$, we have $\left|T_{n}\right|(f)=$ $\sup \left\{|n g| ; g \in L^{\infty}(G),|g| \leq f\right\}=0$. On the other hand, since $n \neq 0$, there exists $g \in L^{\infty}(G)$ such that $\langle n, g\rangle \neq 0$. Now we take $k \in \mathbb{N}$ such that $|g| \leq k 1$. For $\mu \in L^{1}(G)^{+}$, we have

$$
\begin{aligned}
\mu(G)|\langle n, g\rangle| & \leq \mu(G) \sup \left\{|\langle n, h\rangle| ; h \in L^{\infty}(G),|h| \leq k 1\right\} \\
& =\mu(G) k\langle|n|, 1\rangle=k\langle|n| 1, \mu\rangle .
\end{aligned}
$$

Consequently $|n| 1 \neq 0$, i.e. $T_{|n|} \neq 0$.

For $n \in L^{1}(G)^{* *}$, we define $\rho_{n}: L^{1}(G)^{* *} \rightarrow L^{1}(G)^{* *}$ by $\rho_{n}(F)=F n$. The operator $\rho_{n}$ is called a right multiplier on $L^{1}(G)^{* *}$.

Theorem 2.3. Let $n=\Gamma_{E}(\mu)$, where $E$ is a weak*-limit a bounded approximate identity in $L^{1}(G)$ and $\mu \in M(G)$. The following statements hold:

(1) $\left|\rho_{n}\right|(\nu)=\rho_{|n|}(\nu)$, for all $\nu \in L^{1}(G)$.

(2) If $\left|\rho_{n}\right|$ is weak ${ }^{*}$-weak ${ }^{*}$ continuous, then $\left|\rho_{n}\right|=\rho_{|n|}$.

(3) If $m \in L U C(G)^{\perp}$ and $m \neq 0$, then $\left|\rho_{m}\right| \neq \rho_{|m|}$. 
Proof. Since $\mu \in M(G)$, for all $\nu \in L^{1}(G)$, we have $\nu n \in L^{1}(G)$. Hence there exists a measure $\eta \in M(G)$ such that $\nu n=\nu * \eta$ for all $\nu \in L^{1}(G)$ (since $\nu \rightarrow \nu n$ is a right multiplier on $\left.L^{1}(G)\right)$. It is easy to see that $\mu=\eta$.

Now for all $\nu \in L^{1}(G)^{+}$, we can write $\left|\rho_{n}\right|(\nu)=\sup \left\{|F n| ; F \in L^{1}(G)^{* *},|F| \leq \nu\right\}$. But $L^{1}(G)$ is a solid sublattice of $L^{1}(G)^{* *}([5]$, p.234), hence

$$
\begin{aligned}
\left|\rho_{n}\right|(\nu) & =\sup \left\{\left|\eta_{1} n\right| ; \eta_{1} \in L^{1}(G),\left|\eta_{1}\right| \leq \nu\right\} \\
& =\sup \left\{\left|\eta_{1} * \mu\right| ; \eta_{1} \in L^{1}(G),\left|\eta_{1}\right| \leq \nu\right\}=\left|\rho_{\mu}\right|(\nu)=\nu *|\mu| .
\end{aligned}
$$

On the other hand, $\nu *|\mu|=\nu \pi(|n|)=\nu|n|$. Consequently, for all $\nu \in L^{1}(G),\left|\rho_{n}\right|(\nu)=$ $\rho_{|n|}(\nu)$.

2) By (1) and the Goldestines theorem, we have $\left|\rho_{n}\right|=\rho_{|n|}$.

3) If $m \in L U C(G)^{\perp}$, then for all $\nu \in L^{1}(G), \nu m=0$. So for $\mu \in L^{1}(G)^{+}$, we have $\left|\rho_{m}\right|(\mu)=\sup \left\{|\nu m| ; \nu \in L^{1}(G),|\nu| \leq \mu\right\}=0$. By a similar argument as given in part (3) of Proposition 2.2, for all $\mu \in L^{1}(G)^{+}$, we have $\mu|m| \neq 0$, i.e. $\rho_{|m|}(\mu) \neq 0$. Consequently $\left|\rho_{m}\right| \neq \rho_{|m|}$.

Theorem 2.4. Let $G$ be a compact group and $\mu \in L^{1}(G)$. The following statements hold:

(1) $\left\{|P \mu| ; P \in L^{1}(G)^{* *},|P| \leq F\right\}=\left\{|P \mu| ; P \in L^{1}(G)^{* *},|P| \leq E F\right\}$ where $E$ is a weak limit positive approximate identity with norm one in $L^{1}(G)$ and $F \in L^{1}(G)^{* *}$ with $F \geq 0$.

(2) $\left|\rho_{\mu}\right|=\rho_{|\mu|}$.

Proof. Let $F \in L^{1}(G)^{* *}$ and $F \geq 0$. If $P \in L^{1}(G)^{* *}$ and $|P| \leq F$, then $|E P| \leq E F$ and $E P \mu=P \mu$. Indeed, since $G$ is compact, $L^{1}(G)$ is an ideal in $L^{1}(G)^{* *}$ [4], hence $E P_{\mu}=P_{\mu}$. Consequently

$$
\left\{|P \mu| ; P \in L^{1}(G)^{* *},|P| \leq F\right\} \subseteq\left\{|P \mu| ; P \in L^{1}(G)^{* *},|P| \leq E F\right\} .
$$

To prove the reverse inclusion, let $P \in L^{1}(G)^{* *}$ and $|P| \leq E F$. Since $G$ is compact, $\pi(P)$ and $\pi(F)$ are measures in $M(G)$. If $\pi(P)=\nu$ and $\pi(F)=\eta$, then for $f \in C(G)$, $|\langle\nu, f\rangle| \leq\langle\eta,|f|\rangle=\langle F,|f|\rangle$. So we can choose a $P_{1} \in L^{1}(G)^{* *}$ such that $\left|P_{1}\right| \leq F$ and $\langle\nu, f\rangle=\left\langle P_{1}, f\right\rangle$ for all $f \in C(G)$. Hence for $f \in C(G)$, we have $\left\langle P_{1} \mu, f\right\rangle=\left\langle P_{1}, \mu f\right\rangle=$ $\langle\nu, \mu f\rangle=\langle P, \mu f\rangle=\langle P \mu, f\rangle$, i.e. $P \mu=P_{1} \mu$. Consequently,

$$
\left\{|P \mu| ; P \in L^{1}(G)^{* *},|P| \leq F\right\}=\left\{|P \mu| ; P \in L^{1}(G)^{* *},|P| \leq E F\right\} .
$$

2) Let $F \in L^{1}(G)^{* *}, F \geq 0$ and $\pi(F)=\eta$. It is easy to see that

$$
\left\{|P \mu| ; P \in L^{1}(G)^{* *},|P| \leq E F\right\}=\{|\nu * \mu| ; \nu \in M(G),|\nu| \leq \eta\}
$$

So by (1), $\left|\rho_{\mu}\right|(F)=\eta *|\mu|=F|\mu|$. Indeed, since $G$ is compact, $F|\mu| \leq L^{1}(G)$, and any $f \in C(G)$ is of the form $f=g \nu$ for some $g \in L^{\infty}(G)$ and $\nu \in L^{1}(G)$. Hence

$$
\langle F|\mu|, f\rangle=\langle F|\mu|, g \nu\rangle=\langle\nu F|\mu|, g\rangle=\langle\nu * \eta *|\mu|, g\rangle=\langle\eta *|\mu|, g \nu\rangle=\langle\eta *|\mu|, f\rangle .
$$


Consequently $\rho_{|\mu|}=\left|\rho_{\mu}\right|$.

For $n \in L^{1}(G)^{* *}$, we denote $\lambda_{n}$ as a left multiplier on $L^{1}(G)^{* *}$. We know that $\left|\lambda_{n}\right|$ is a left multiplier if and only if $\left|\lambda_{n}\right|=\lambda_{|n|}$ ([3], Lemma 3.6). For $n \in L U C(G)^{\perp},\left|\rho_{n}\right|(\nu)=0$ $\left(\nu \in L^{1}(G)\right)$. If $\left|\rho_{n}\right|$ is a right multiplier, then $\left|\rho_{n}\right|$ is weak*-weak* continuous, so $\left|\rho_{n}\right|=0$. Moreover, if $n \neq 0$, then $\rho_{|n|} \neq 0$, i.e. $\left|\rho_{n}\right| \neq \rho_{|n|}$. Also, it is not known whether for any $\mu \in M(G)$, we have $\left|\rho_{\mu}^{* *}\right|=\rho_{|\mu|}{ }^{* *}$. In the following Corollary we give some cases where the equality holds.

Corollary 2.5. For $\mu \in L^{1}(G),\left|\rho_{\mu}^{* *}\right|=\rho_{|\mu|}{ }^{* *}$ whenever one of the following conditions holds:

(1) $G$ is a compact group.

(2) $\left|\rho_{\mu}^{* *}\right|$ is compact.

(3) $\left|\rho_{\mu}^{* *}\right|$ is weak ${ }^{*}$-weak* continuous.

Proof. Assume that (1) holds. By Theorem 2.4, $\left|\rho_{\mu}^{* *}\right|=\left.\rho_{|\mu|}\right|^{* *}$. If (2) holds, since $\left|\rho_{\mu}^{* *}\right|$ is compact, so $\left|\rho_{\mu}\right|: L^{1}(G) \rightarrow L^{1}(G)$ is compact. Consequently $G$ is compact [7]. The statement follows from (1). Suppose (3) holds. By Theorem 2.3, $\left|\rho_{\mu}^{* *}\right|=\rho_{|\mu|}{ }^{* *}$.

\section{Acknowledgement}

This work has been done while the author was visiting The Department of Mathematical Science of Alberta University. The author would like to thank both Professor A. T. Lau and University of Alberta for their hospitality.

\section{References}

[1] C. D. Aliprantis and O. Burkinshaw, Positive Operators, Academic Press, New York/London, 1985.

[2] F. Ghahramani and A. T. Lau, Multipliers and ideals in second conjugate algebras related to locally compact groups, J. Funct. Anal. 132(1995), 170-191.

[3] F. Ghahramani and A. T. Lau, Multipliers and modulus on Banach algebras related to locally compact groups, J. Funct. Anal. 150(1997), 478-497.

[4] M. Grosser, $L^{1}(G)$ as an ideal in its second dual space, Proc. Amer. Math. Soc. 73(1979), 363-364.

[5] J. L. Kelly and I. Namioka, Linear Topological Spaces, Van Nostrand, Prinston, NJ, 1963.

[6] A. T. Lau and J. S. Pym, Concerning the second dual of the group algebra of a locally compact group, J. London. Math. Soc. 41(1990), 445-460.

[7] S. Sakai, Weakly compact operators on operator algebras, Pacific J. Math. 14(1964), 659664.

Department of Mathematics, Tarbiat Modarres University, P.O.Box 14115/175, Tehran, Iran. 